\title{
Overcurrent Protections in MV Grid with Local Energy Sources
}

\author{
Józef Lorenc , Bartosz Olejnik, and Aleksandra Schött \\ Institute of Electrical Power Engineering, Poznań University of Technology, Poznań, Poland
}

\begin{abstract}
The article presents typical methods of connecting various types of local power sources to a medium voltage network. Describes the advantages and disadvantages of individual solutions. The authors focus particularly on the issues of power system protection, with particular emphasis on overcurrent protection (ANSI 50 and 67). The key part of the article is the presentation of the selection methodology, along with examples, of setting this type of protection for a frequently encountered case - connecting local energy sources with busbars with a line with receipts.
\end{abstract}

\section{Introduction}

The article applies only to networks with local power sources connected to medium voltage lines derived from $\mathrm{HV} / \mathrm{MS}$ stations, whose main task is to supply consumers, and the value of power absorbed from the $110 \mathrm{kV}$ network is usually much higher than the power generated by local sources (the local power plant designation LPP will be used later in the text). The properties of such systems do not depend on the type of primary energy used (water, wind, biomass), but on the type of generators and network parameters.

The principles given in this article should be treated as a proposal, and the substantive value should be assessed independently. It seems that only exploitation will show the superiority of some theories after the failure of the network with LPP and their correct or incorrect liquidation. An additional difficulty is often poor recognition of the automation installed in the LPP itself due to the lack of data or deliberate operation of the manufacturer.

Relay settings in networks with LPP are a big problem for most engineers dealing with power system protection. In this publication, the author's methodology for selecting the overcurrent protection settings in Poland will be presented. It is noted that due to different network configurations, the dependencies given here may not be appropriate for cases in other countries.

Due to the author's approach to the topic, the number of publications cited is small.

\section{Types of local energy sources and their connection methods}

From the point of view of the type of generators, the following division can be made [1,2]:

- synchronous generators working synchronously with the network, i.e. connected directly to the network (without rectifying-wave circuits) - the most dangerous, because they cause an increase in short-circuiting powers and the danger of maintaining the so-called load island,

- asynchronous generators - less dangerous, but still generate short-circuit current - usually in a relatively short time, because they basically require cooperation with a source of passive power,

- synchronous generators working asynchronously with the network, i.e. indirectly connected (with rectifying-wave systems) - the most safe, but rather found in large wind farms, the article does not apply,

- DC power sources with inverters, i.e. photovoltaic sources, just as safe as listed in point $\mathrm{c}$ and this article is not considered.

Regarding the sources given in point $b$, it should be noted that various behaviors are possible. In experimental studies, in which Poznan University of Technology participated, it was shown that in such systems shortcircuit current can be generated even for a few seconds.

In this work, only connections according to the diagrams given in Figures 1 and 2 will be considered. In Figure 1 LPP are connected to the line with receivers, i.e. $\mathrm{MV} / \mathrm{LV}$ stations. The connection method can be very different, in Fig.1a there is one power plant with one common transformer and several generators (this is often the case for hydroelectric plants), and in Fig.1b several power plants connected in different places of the line (this may be for small wind farms) ). White rectangles mean "protection point" hereinafter referred to as PP, which consists at least of transformers (CT, without/and/or VT), protection and circuit breaker.

In Figure 2, LPPs are connected to the so-called subscriber line, it has no load. There may be systems that differ from the number of connected sources or other details, but these differences will not affect the overall distribution.

Equipment with power protection can also be different. For example - for the system in Fig. 1a just 
before the PP-E protection point from the line side, a remotely controlled recloser may be installed [3], which is also equipped with power protections. In the same example, there is no need to be a PP-T security point.
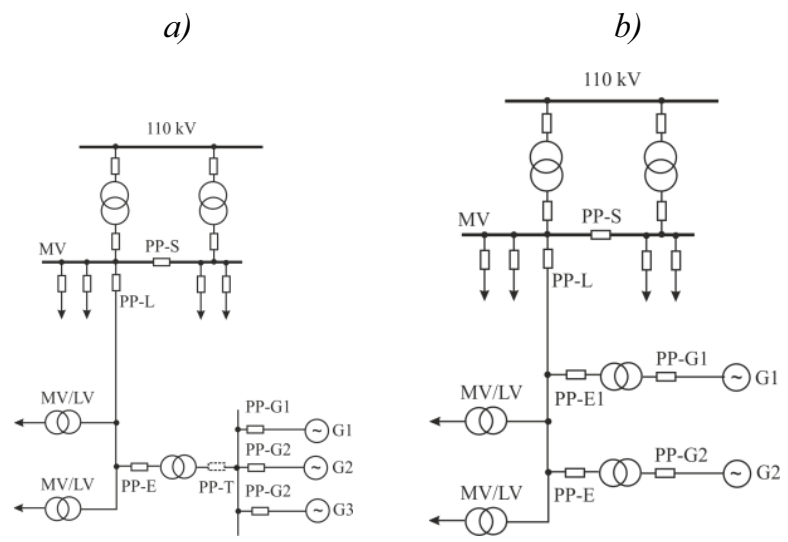

Fig. 1. Connection of LPP to the line with receipts. Explanations for Figures 1 and 2: PP-L - the protection point of the line in the transformer-switch station, PP-S - trunk connector, PP-E - local power plant, PP-G - generator. a)

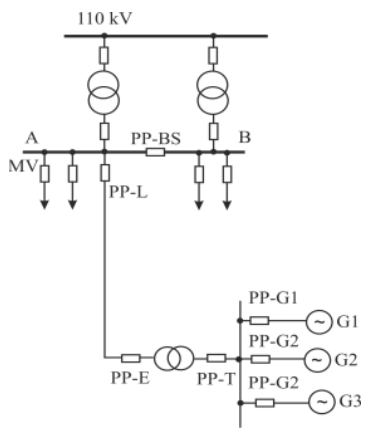

b)

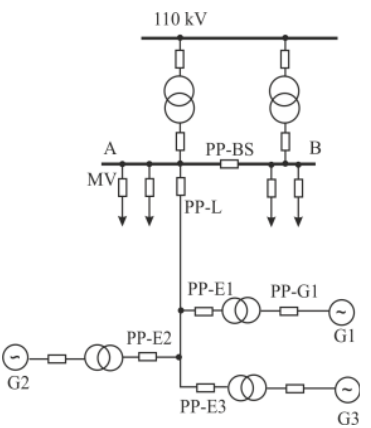

Fig. 2. Connection to the subscriber line.

\section{General requirements}

Power system protection systems in LPP networks put the basic requirements, i.e. selectivity and discrimination, speed and reliability of operation, sensitivity, economy, i.e. the same as typical systems for classic networks. In addition, they also put up some specific requirements:

- enabling the execution of a correct autoreclosure (ARC) cycle in nearby overhead lines,

- enabling the execution of the automatic transfer switching (ATS) cycle between the $110 \mathrm{kV} / \mathrm{SN}$ station sections,

- $\quad$ correct operation of busbar protection (BP) and local circuit breaker (LCBR) reserve in HV/MV station,

- protection against the effects of island work (especially in terms of lowering or increasing voltage or the quality of electricity).

During the analysis, the assumption was made that the fields in the HV/MV substation are equipped with typical security and automation: ARC, ATS, automatic load shelding, LCBR and overcurrent logic busbar protection. Short-circuit and short-circuit overcurrents are used against the effects of phase-to-phase short- circuits, and they are based on zero current and voltage components and their associated quantities.

Depending on the technical details, including the type of protections used, but also their settings, there may be the following risks from local power plants [4]:

- creation of a load island with improper electricity parameters; the increase of electric voltage is particularly dangerous,

- the possibility of unnecessary line disconnections with local energy sources during short circuits in other parts of the network,

- obstructing ARC automation by supplying voltage and burning of an electric arc,

- obstructing the implementation of the ATS automation cycle, if the MV switchgear section is switched,

- unnecessary blocking of busbar protection during short-circuits on busbars,

- difficulties in implementing the LCBR.

In this work, the principles of selection of overcurrent protections and their settings for networks with lines to which both receipts and LPP are connected are presented.

\section{Phase-to-phase short-circuits in the MV network}

\subsection{Overcurrent protections}

The risk assessment and some suggestions regarding the possibility of avoiding them during phase-to-phase shortcircuits will be carried out on the basis of the diagram shown in Figure 3. The short circuits for which the analysis will be carried out are marked with symbols from $\mathrm{K} 1$ to $\mathrm{K} 4$. In each of these short-circuits shortcircuit current is the result of superposition of two components: flowing from the power system side $\left(I_{k}^{S}\right)$ and from the LPP side $\left(I_{k}^{E}\right)$.

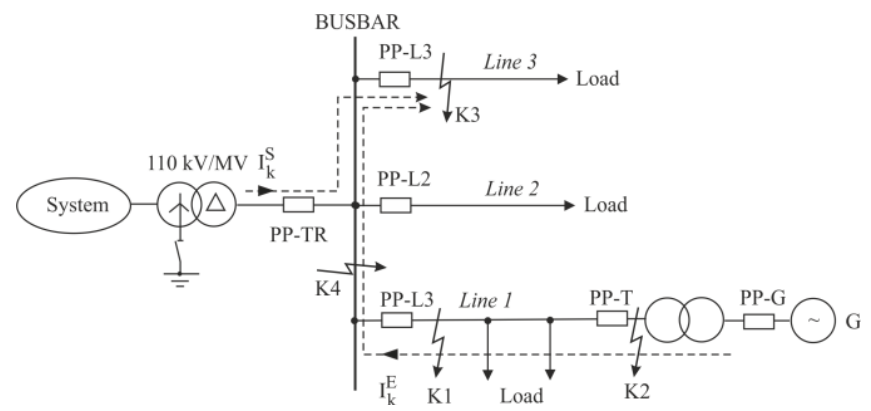

Fig. 3. Operation of overcurrent protection against the effects of phase-to-phase faults.

It is proposed to use directional blocks and exemplary time settings according to the proposal shown in Fig. 4, where each short circuit in the line and LPP will be switched off selectively. At points PP-L3, PP-T and PP$\mathrm{G}$, two delayed-current protection are proposed: one with a longer time without directional blocking (ANSI 50) and the other with a shorter time, with a directional block (ANSI 67) [5,6]. The problem may be to provide directional blocks for overcurrent protection. IEDs are 
generally equipped with this function, but not always in every place of the network there are voltage transformers necessary for its proper operation

\section{BUSBAR}

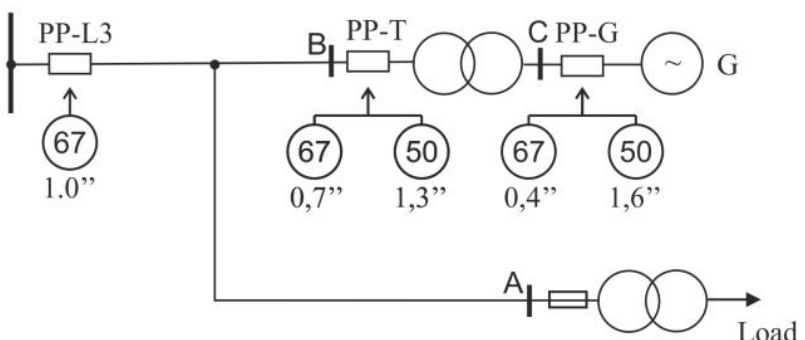

Fig. 4. Proposal of selecting delayed overcurrent protection $(50 / 67)$ for the line with generation and consumers.

At point PP-L3, the current setting value should be selected according to the following:

$$
I_{\text {set }} \geq \frac{k_{b} k_{r} I_{\max }}{k_{p} \vartheta_{i}}
$$

where:

- $I_{\text {nast }}$ - current setting of the protection,

- $\quad k_{\mathrm{b}}$ - a safety factor that can be taken equal to 1.1 1.2 ,

- $\quad k_{\mathrm{r}}$ - coefficient of self-starting of the motors supplied from the protected section of the network, depending on the content of the motor load in the overall load, the range of its value is $1-4$,

- $\quad I_{\max }$ - the maximum load current of the protected line segment, depending on the LPP power, it may be the current resulting from its rated power or the current resulting from the load parameters, for example inactive LPP,

- $k_{\mathrm{p}}$ - resetting ratio,

- $\vartheta_{\mathrm{i}}$-current transformer ratio.

Sensitivity should be checked according to (2), where:

- $\quad I_{\mathrm{kmin}}$ - the minimum current flowing through the protection point during a short circuit at the end of the protected section (it is always a two-phase short-circuit current for the network system when it is the smallest - eg in summer),

- $\quad k_{\mathrm{c}}$ - sensitivity factor which must be taken equal 1.5 for the basic protection, and 1.2 for the reserve protection.

$$
I_{\text {set }}<\frac{I_{k m i n}}{k_{c} \vartheta_{i}}
$$

Sensitivity should be checked for the following cases:

- in places $\mathrm{A}$ and $\mathrm{B}$ with the required $\mathrm{kc}=1,5$,

- in place $\mathrm{C}$ at $\mathrm{kc}=1,2$ (may be a problem with this requirement).

For PP-T and PP-G security you should use (1) and check the sensitivity in (2). For PP-T it is necessary to check the sensitivity for point A taking into account the current flowing only from the $\mathrm{G}$ side and for shorting on the busbars $110 \mathrm{kV} / \mathrm{MV}$ at $k_{\mathrm{c}}=1.5$ and for point $\mathrm{C}$ taking into account the current flowing from the power system side also at $k_{\mathrm{c}}=1.5$. For PZ-T, check the sensitivity for short-circuit in $\mathrm{G}$ considering the short-circuit current flowing from the system, and in points $\mathrm{C}$ and $\mathrm{B}$ from the side $\mathrm{G}$.

If near PP-T, the so-called recloser, it will not be possible to get selectivity, because there will be no time grading [5]. You can increase the time delay in PPL-3 to, for example, 1.3 or $1.5 \mathrm{~s}$ and get this option. It seems that both the recloser and the PP-T security can be set the same way and do not require selectivity between these points, because the physical distance between them will be very small. The task of the recloser is usually to obtain the ability to remotely disable LPP by the disposition without interfering with devices not owned by the network operator [7].

BUSBAR

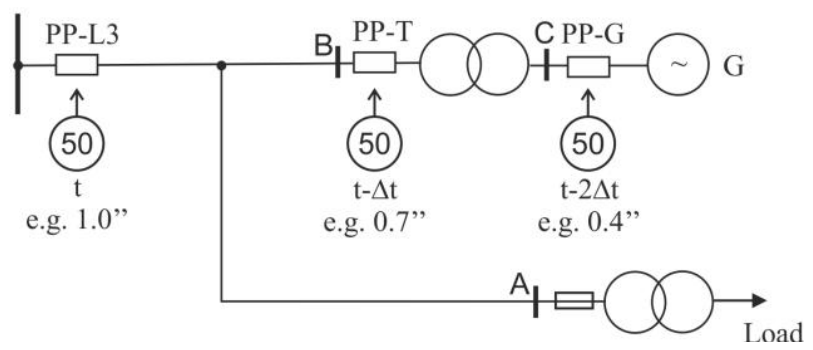

Fig. 5. Simplified variant of the selection of delayed overcurrent protections (50) and their time settings for the line with loads.

If it is not possible to use directional interlocks, it is suggested to select the protections as in Fig.5. At the same time, more general expressions were given to the selection of the time delay. Grading started from the setting in the linear field of the switchboard, because as already mentioned, increasing this setting is extremely difficult and inadvisable. The given values are just an example. In this situation, any phase-to-phase fault in the cooperating MV network may result in the LPP being turned off. The setting values of the currents should be selected in accordance with (1) and (2). This is not a solution consistent with the classic principles of protection automation, because it has no selectivity, but it is simple and the short-circuit will be turned off. It should be anticipated that in the case of a phase-to-phase shortcircuit in another line, the LPP will first be switched off, and only then the damaged line will be disconnected.

\subsection{Short-circiut protections $(50)$}

Short-circuit protection is required in PPL-3 for the line and at least in PP-T for the power plant transformer.

The short-circuit protection in PPL-3 should be set so that its range ends before the PP-T point. This requirement can be achieved by choosing the setting according to the formula

$$
I_{s e t} \geq \frac{k_{b} I_{k \max }}{\vartheta_{i}}
$$

where: 
- $\quad k_{\mathrm{b}}$ - safety factor, selected from 1.2 to 1.6 ,

- $I_{\mathrm{kmax}}$ - maximum short-circuit current flowing through the PPL-3 from the power system side during short-circuit near PP-T, it will be a threephase short-circuit current at the highest shortcircuit power on the $110 \mathrm{kV} / \mathrm{MV}$ station busbars.

The time delay of this protection can be in the range of 0.1-0.3 s, it is difficult to indicate a clear selection rule. To obtain the maximum value of this time, the heat resistance of overhead lines is sometimes calculated.

It is also necessary to check if in case of shorting on busbars current flowing to them from the $\mathrm{G}$ side will not trigger the short-circuit protection at point PP-L3. It is not about the missing operation during shorting on busbars, because it will lead to switching off the LPP, but during short circuits at the beginning of the other lines led out from the $110 \mathrm{kV} / \mathrm{SN}$ station. Therefore, the dependence (3) should be checked when inserting as $I_{\mathrm{kmax}}$ the value of the maximum short-circuit current flowing from the LPP side when shorted on busbars. If the requirement is not met, which is rare, use the directional interlock (ANSI 67) for the short-circuit protection at PPL-3. Failure to meet the requirements will only occur if the current flowing from the LPP side is greater by at least $20 \%$ higher than the current flowing from the power system side when shorted on busbars. The occurrence of this condition is possible with a small electrical distance LPP from busbars and high power (on the order of several MW).

The short-circuit protection of the transformer at the PP-T point, which is its protection during internal faults, should be set according to the higher value obtained from two dependencies:

$$
\begin{gathered}
I_{\text {set }} \geq \frac{k_{b}^{\prime} I_{n T}}{k_{p} \vartheta_{i}} \\
I_{\text {set }} \geq \frac{k_{b}^{\prime \prime} I_{\text {kmax }}}{k_{p} \vartheta_{i}}
\end{gathered}
$$

in which:

- $\quad I_{\mathrm{nT}}-$ transformer rated current,

- $I_{\mathrm{kmax}}$ - maximum short-circuit current on rails behind the transformer,

- $\quad k_{\mathrm{b}}{ }^{\prime}$ - safety factor including the impact of the magnetizing current at switching the transformer to idling $(=4-8)$,

- $\quad k_{\mathrm{b}}$ " - safety factor for resetting the current setting of this protection from the delayed protections located behind the transformer, i.e. practically in PP-G (= 1.2-1.6).

Dependence (5) is to protect the transformer from unnecessary disconnections from the impact of the magnetizing current. It is assumed that it is energized only from the network side and the generator (s) are synchronized to it. The value of the coefficient is dependent on the time delay, which should be within 0.1 - $0.3 \mathrm{~s}$, the longer the time, the coefficient may be smaller.

If the system does not have a directional block for delayed overcurrent protections, the principle of selecting short-circuit protection does not change.

\section{Summary}

The article presents typical LPP connection systems with the medium voltage network used in Poland, also giving the advantages and disadvantages of specific solutions. It seems that in other countries, the problem of connecting LPP, especially with relatively low power (up to several MW), will be based on similar assumptions.

Due to limitations in the publication, the focus was only on the problem of setting the overcurrent and shortcircuit overcurrent protection (ANSI 50), also with the directional components (ANSI 67).

The connection of LPP to the MV line and switchgear carries many threats to the correct operation of power system protection in the cooperating network. The article presents a few of these threats, of which it is necessary to be aware.

When selecting protection and their settings, special attention should be paid to the protection of recipients. It seems that the worst disruption would be an increase in voltage that can cause damage - especially electronic equipment.

In relation to delayed overcurrent protection in MV networks, the principle should be formulated that in the case of using digital constructions (terminals or field controllers) it is recommended to graduate temporarily every $0.3 \mathrm{~s}$, and not as for older electromechanical or static analog solutions $-0.5 \mathrm{~s}$. This principle is not related to the existence of the LPP network, but has a high degree of generality. Its introduction will shorten the duration of phase-to-phase faults, and sometimes improve the selectivity of security in the network.

The conclusion from the main thread of the article is the thesis that the connection of a local power plant to the line with the recipients worsens the conditions of their supply, especially by increasing the probability of interruptions in energy supply.

\section{References}

1. J. Martínez, P. C. Kjær, P. Rodriguez and R. Teodorescu, "Short circuit signatures from different wind turbine generator types," 2011 IEEE/PES Power Systems Conference and Exposition, Phoenix, AZ, 2011, pp. 1-7.

2. E. Muljadi, N. Samaan, V. Gevorgian, Jun Li and S. Pasupulati, "Short circuit current contribution for different wind turbine generator types," IEEE PES General Meeting, Minneapolis, MN, 2010, pp. 1-8.

3. J. Lorenc, J. Andruszkiewicz, B. Olejnik, B. Staszak and P. Balcerek, "Earth fault detection and isolation system for MV network," 2015 Modern Electric Power Systems (MEPS), Wroclaw, 2015, pp. 1-7.

4. T. Gjengedal, "Integration of wind power and the impact on power system operation," Large Engineering Systems Conference on Power Engineering, 2003, 2003, pp. 76-83.

5. IEEE Standard Electrical Power System Device Function Numbers, Acronyms, and Contact Designations," in IEEE Std C37.2-2008 (Revision of 
IEEE Std C37.2-1996), vol., no., pp.1-48, Oct. 3 2008.

6. S. A. Gopalan, V. Sreeram, H. H. C. Iu, "A review of coordination strategies and protection schemes for microgrids", Renewable and Sustainable Energy Reviews, vol. 32, pp. 222-228, 2014.
7. P. I. Santos e Abreu and A. G. Martins, "Assessment of the behavior of protection systems in radial networks with distributed generation," 2016 51st International Universities Power Engineering Conference (UPEC), Coimbra, 2016, pp. 1-6. 